\title{
MATHEMATICAL PROGRAMMING COMO FERRAMENTA GERENCIAL EM MICRO E PEQUENAS EMPRESAS: UMA PROPOSTA PARA APOIO À DECISÃO NO SETOR DE VESTUÁRIO
}

\section{MATHEMATICAL PROGRAMMING AS A MANAGEMENT RESOURCE IN MICRO AND SMALL ENTERPRISES: A PROPOSAL TO SUPPORT DECISIONS IN THE APPAREL SECTOR}

\section{$\underline{\text { Diego Vieira dos Santos }}{ }^{1}$, Keydson Quaresma Gomes ${ }^{2}$, Alessandro Roberto Rocha ${ }^{3}$,

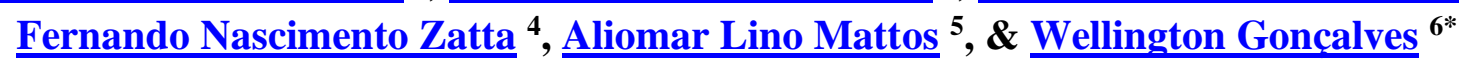

${ }^{1}$ Programa de Gestão Pública, Universidade Federal Espírito Santo (UFES). ${ }^{2}$ Centro Universitário Norte do Espírito Santo (CEUNES/UFES). ${ }^{3}$ Instituto de Ciências da Vida, Universidade Federal de Juiz de Fora (UFJF). ${ }^{4}$ Centro de Ciências Sociais Aplicadas, Universidade Presbiteriana Mackenzie. ${ }^{5}$ Centro de Ciências Jurídicas e Econômicas (CCJE/UFES). ${ }^{6}$ Laboratório de Pesquisa Operacional, Logística e Transportes (POLT) (CEUNES/UFES). ${ }^{1}$ diegovs.89@ gmail.com ${ }^{2}$ keydson.gomes@ufes.br ${ }^{3}$ alessandro.rocha@ufjf.edu.br ${ }^{4}$ zatta@hmzconsulting.com.br ${ }^{5}$ aliomargstones@ hotmail.com ${ }^{6 *}$ wellington.goncalves@ufes.br

\section{ARTIGO INFO.}

Recebido em: 12.05.2021

Aprovado em: 30.06.2021

Disponibilizado em: 13.07.2021

\section{Palavras-chave:}

Previsão de negócios; Otimização de processos; Gestão de custos; Análise de tendências.

\section{KEYWORDS:}

Business forecasting; Optimization of processes; Cost management; Trend analysis.

\section{*Autor Correspondente: Gonçalves, W. \\ RESUMO}

Em um ambiente de instabilidade econômica a busca de estratégias que garantam a subsistência do empreendimento tem impulsionado as Micro e Pequenas Empresas (MPE's) no Brasil a assumirem uma postura mais ágil nas tomadas de decisão. Assim, o presente estudo teve como objetivo a elaboração de uma proposta para auxiliar a tomada de decisão voltada à gestão de produção e operações - de uma empresa do setor de vestuário utilizando-se de Linear Programming (LP). A coleta de dados e aplicação da metodologia foi direcionada a uma MPE localizada na região do Vale do Rio Doce (Minas Gerais). O desenvolvimento da solução ocorreu por meio de linguagem de programação primária. Os resultados permitiram, além de subsídios para decisões, parametrizar a solução para necessidades cotidianas. O modelo determinístico apresentado neste caso se mostrou uma ferramenta eficaz para lidar com questões de gerência empresarial e de produção, sobretudo com relação a alocações ótimas de recursos, o qual pode e deve ser adaptado para diferentes cenários e condições.

\begin{abstract}
In an environment of economic instability, the search for strategies that guarantee the enterprise survivor has driven Micro and Small Enterprises (SMEs) in Brazil to assume a more agile posture in decisionmaking. Thus, this study aimed to prepare a proposal to assist decision making-focused on production and operations management - of a company in the apparel sector using Linear Programming (LP). Data collection and application of the methodology were directed to an MPE located in the region of Vale do Rio Doce (Minas Gerais). The development of the solution took place through the primary programming language. The results allowed, in addition to subsidizing decisions, parameterizing the solution for everyday needs. The deterministic model presented in this case proved to be an effective tool to deal with business and production management issues, especially with regard to optimal resource allocation, which can and should be adapted to different scenarios and conditions.
\end{abstract}




\section{INTRODUÇÃO}

O funcionamento de entidades empresariais em cenários de instabilidade econômica requer o guarnecimento de soluções emergentes, adaptáveis ao modelo de gestão, e que possam continuamente, subsidiar gestores em prol da implementação de ações capazes de promover competitividade aos negócios (Silva et al., 2020; Turra et al., 2017).

A obtenção de vantagem competitiva, por sua vez, consubstancia-se com a adoção de estratégias que estabelecem diferenciação entre as organizações em seu respectivo segmento de atuação e, dentro deste contexto, a habilidade em criar valor junto aos stakeholders tem sido ancorada, principalmente, na capacidade dos empreendimentos em assumirem liderança em custo (Hamidi et al., 2019; Brito \& Brito, 2012; Porter, 1996).

Sob esta perspectiva, efeitos do avanço tecnológico contemporâneo têm se mostrado oportunos no sentido de impulsionar a competitividade entre as organizações, a partir do aprimoramento e aplicabilidade de técnicas e ferramentas de gestão que ensejam maior assertividade nas tomadas de decisão e, por conseguinte, convergem em favor da alavancagem e longevidade dos negócios (Serviço Brasileiro de Apoio às Micro e Pequenas Empresas [SEBRAE], 2014).

No segmento empresarial de médio e grande porte, desdobramentos da instabilidade externa se mostram menos impactantes do que nas Micros e Pequenas Empresas (MPE's), afirma Birbirenko (2020). Esta autora explica que, fatores como: suficiência de recursos, capacidade de adaptações ágeis às transformações do mercado, previsão de desenvolvimento futuro, e redução de riscos dos negócios por meio da diversificação do mercado, colocam os grandes empreendimentos à frente dos pequenos negócios.

À vista disto, a instauração de estratégias competitivas ainda se apresenta incipiente no âmbito das MPE's brasileiras, suscitando vulnerabilidade e efemeridade neste profuso segmento, responsável por movimentar $27 \%$ do PIB e mais de $50 \%$ da empregabilidade formal no país (SEBRAE, 2020; Pereira et al., 2019).

Frente a este cenário, parâmetros de suporte à decisão baseados em modelagem matemática emergem como ferramentas de gestão subsidiando o delineamento de ações pelos executivos, a partir de representações operacionais (Maiti et al., 2020; Santos \& Vallim, 2020). Assim, é possível obter informações mais precisas, bem como realizar previsões com elevada acurácia e estimativas de risco.

Nesse sentido, a Linear Programming (LP) desponta como a área da Pesquisa Operacional que contribui no processo decisório e, além de se destacar pela ampla aplicabilidade, figura como notória ferramenta de gestão ao oferecer fidedignidade às informações geradas, e propiciar maior assertividade pelos gestores nas tomadas de decisão frente às MPE's (Santos \& Vallim, 2020; Freddi, 2019).

Diante do exposto, este estudo de caso teve por objetivo a elaboração de uma proposta para auxiliar a tomada de decisão em uma empresa do setor de vestuário, fundamentando-se na modelagem matemática por meio do solver LINGO. Para tanto, foi empregada uma abordagem metodológica que considerou inteirações com seus pares e o mercado, a partir de um survey e

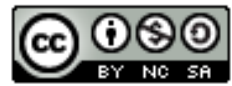


Citação (APA): Santos., D. V., Gomes, K. Q., Rocha, A. R., Zatta, F. N., Mattos, A. L., \& Goncalves, W. (2021). Mathematical Programming como ferramenta gerencial em micro e pequenas empresas: uma proposta para apoio à decisão no setor de vestuário. Brazilian Journal of Production Engineering, 7(3): 56-70.

da utilização da LP. Os resultados obtidos foram comparados com os do atual sistema de custeio da empresa, e, a partir disso, foram apresentadas as contribuições da aplicação de uma reflexão que é estendida a demais empresas de pequeno porte, assim como aos stakeholders e mercado.

\section{REFERENCIAL TEÓRICO}

\subsection{O Setor Confecção e os Desafios De SubsistênCia na ConTEMPoraneidade}

De acordo com a Confederação Nacional da Indústria (CNI, 2017), o setor de vestuário e de confecção é um dos setores industriais mais tradicionais e dinâmicos do mundo. A entidade ressalta que as indústrias desse setor apresentam uma extensa cadeia produtiva, englobando desde a etapa de produção de fibras até a etapa de confecção, e estão presentes nas mais diversas regiões do planeta.

No Brasil, o setor é responsável por cerca de 1,5 milhão de empregos diretos e $6 \%$ do faturamento da indústria de transformação, contando com mais de 32 mil empresas que são formadas majoritariamente (96,8\%) por Micro e Pequenas Empresas (MPE's) (CNI, 2017). Em complemento, Maciel et al. (2019) mencionam que o país se notabiliza por ser "a última cadeia de vestuário completa do Ocidente", uma vez que abrange desde a produção de fibras, passando pela etapa de fiação, tecelagem, confecção, varejo e até desfiles de moda.

Em particular, o setor de confecção possui o quarto maior parque produtivo do mundo e absorve em torno de $75 \%$ da força de trabalho de toda a indústria de vestuário e de confecção no país (CNI, 2017). No ano de 2017, a indústria de confecção atingiu 5,9 bilhões de itens produzidos (Maciel et al., 2019).

Gamboa et al. (2020) acrescentam que a etapa da confecção de vestuários registra um elevado número de pequenas empresas, com considerável grau de informalidade e perda da capacidade produtiva decorrentes da ausência de ganhos de escala, sendo que um dos fatores que contribuem para essa situação refere-se, na visão desses autores, a aspectos do regime tributário predominante no setor.

As empresas nacionais da indústria de confecção, segundo Godinho e Corso (2019), tiveram ápice de produção até o final da década de 1970, mas, a partir da década de 1990, essas empresas acabaram perdendo participação no mercado, principalmente com o estímulo no país ao comércio internacional e a concorrência de empresas asiáticas.

No século XXI, Filleti e Boldrin (2020) mencionam que o setor de vestuário vem percebendo uma tendência de desindustrialização no Brasil. As autoras atribuem a esse fenômeno a manutenção de expressivos custos operacionais, declínio nas taxas de investimento para o setor, e apreciação nas taxas de câmbio, fator este que dificulta a competitividade das indústrias nacionais frente ao mercado global. Segundo o relatório da Associação Brasileira da Indústria Têxtil e de Confecção (ABIT, 2021) sobre o nível de atividade do setor, observa-se queda no volume de exportações no primeiro trimestre de 2021, se comparado ao mesmo período de 2020 (Figura 1).

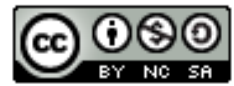


Figura 1. Extrato do comportamento da balança comercial do setor de vestuário

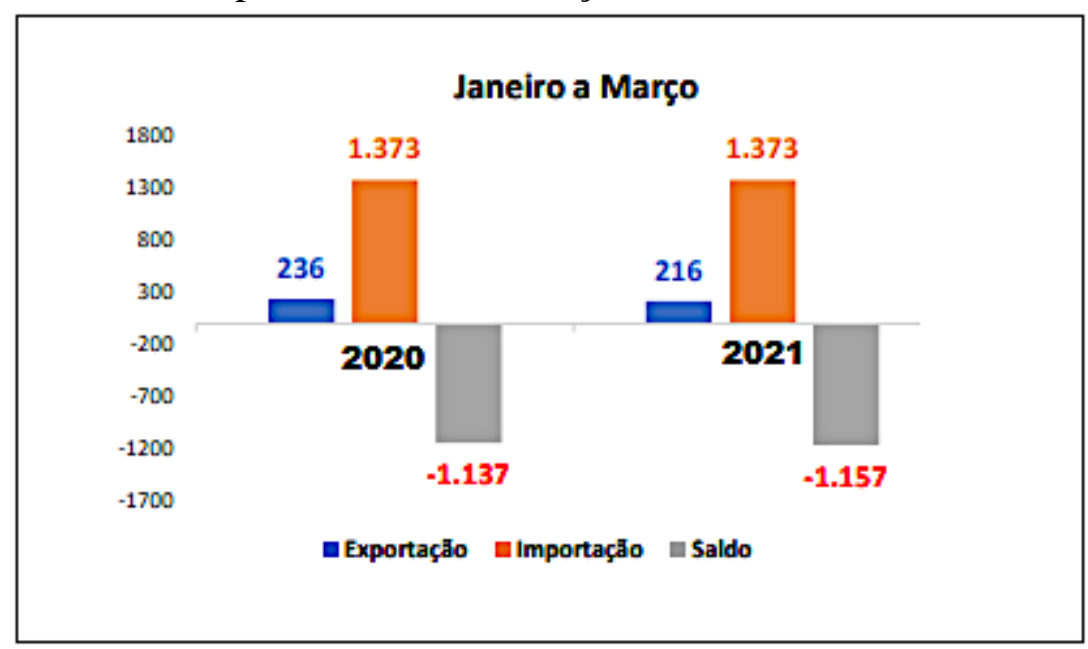

Nota: Produtos sem fibra de algodão, em US\$ (milhões).

Fonte: ABIT (2021).

Nesse sentido, além de enfrentar os desafios pertinentes ao enquadramento legislativo, especialmente quanto a questões tributárias, às inovações tecnológicas, à concorrência internacional, entre outros, o setor tem enfrentado as consequências da pandemia do novo Coronavírus (Covid-19). No entendimento de Vasconcelos e Vasconcelos (2020), as medidas de isolamento social adotadas para controle de contágio dessa doença restringem a circulação de trabalhadores e consumidores, o que compromete tanto a demanda por produtos quanto a oferta de trabalho, instaurando uma crise sanitária, social e econômica.

Ainda segundo dados da ABIT, pesquisa realizada entre os dias 23 e 25 de março de 2020 com 225 empresas do ramo concluiu que a pandemia do Covid-19 afetou 97\% dessas empresas por diversos motivos: cancelamento ou adiamento de pedidos de clientes, problemas no escoamento da produção dos clientes, problemas no abastecimento de insumos, alterações nos custos de insumos de produção, entre outros (ABIT, 2020).

Diante desse contexto excepcional, o Instituto de Pesquisa Econômica Aplicada (IPEA, 2020) cita que têm sido implementadas ações governamentais para minimizar os impactos da pandemia no funcionamento e na empregabilidade dos pequenos negócios, tais como a vigência da Resolução n. 850/2020 do Conselho Deliberativo do Fundo de Amparo ao Trabalhador (CODEFAT), que institui linhas de crédito para empresas com faturamento de até $\mathrm{R} \$ 10$ milhões com condições especiais para MPE's; além das Medidas Provisórias nº 936/2020 e nº. 944/2020 que dispõem sobre os aspectos de manutenção do emprego e da renda.

Sendo assim, as MPE's do setor de confecções devem buscar alternativas que possam gerar, entre outras vantagens, o aumento da produtividade e a redução de custos para o alcance da maximização de lucros. No entanto, estudo de Pavão e Camacho (2018) sobre estratégias adotadas por indústrias do ramo, revelou que medidas relacionadas à liderança em custos apresentaram baixos indicadores, e concluiu que as estratégias das confecções estão mais voltadas à diferenciação de produtos. Além disto, Gamboa et al. (2020) mencionam que, a

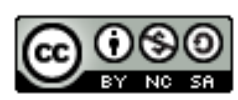


tecnologia consideravelmente estável e a baixa qualificação de mão de obra figuram como desafios relacionados à produtividade para o setor.

Para Siqueira et al. (2019), as empresas devem priorizar as boas práticas de gestão, considerando diversas variáveis, tanto objetivas quanto subjetivas, demonstrando conhecimento integral acerca da demanda de seus produtos no mercado, no intuito de alcançar lucratividade e sustentabilidade a longo prazo. Nesse sentido, podem ser adotados novos padrões de produção, nos quais se promovam a utilização eficiente de recursos e a minimização de perdas e desperdícios (CNI, 2017).

A fim de alcançar os objetivos organizacionais, Gomes et al. (2019) sugerem a utilização de técnicas que envolvam a programação linear, considerada uma das ferramentas mais eficazes no planejamento e acompanhamento de atividades, a qual se baseia em problemas de otimização com múltiplas variáveis de decisão.

\section{2. mathematical Programming como Ferramenta Gerencial em MPE'S}

A necessidade de organizar e otimizar recursos, processos e posicionamento de mercado faz parte das necessidades gerenciais do cotidiano de Micro e Pequenas Empresas (MPE’s). E isso, em diversos casos, significa manter o seu market share o mais estável possível ou, até mesmo, estar operando de maneira sustentável.

Dentro desse contexto, Kapitonov et al. (2017) destacam que as MPE's por suas características necessitam, em sua maioria, de soluções operacionais que sejam efetivas. No entanto, estes autores também enfatizam que tais soluções sejam replicáveis considerando o perfil das MPE's.

Além do reconhecimento da marca no setor de confecção, para Mohd et al. (2017) a lealdade do cliente é desenvolvida a longo prazo a partir de uma confiança conquistada no produto. Por conseguinte, essa confiança começa com a qualidade ofertada, a qual se deve à diferenciação competitiva em termos de preços, volumes de vendas, economias de escala e custos otimizados, concluem estes autores.

Segundo Akaeze (2016), as MPE's criam dois em cada três novos empregos e, produzem duas vezes e meia mais inovações do que as grandes empresas. Para este autor, esse fato se deve a renovação de estratégias utilizadas, quais sejam, em termos gerenciais ou operacionais. Em seu estudo, o autor aponta que estas empresas, ao utilizarem ferramentas computacionais, apresentam resultados favoráveis e, em contrapartida, aquelas que possuem planejamentos e ações manuais possuem limitado market share no mercado.

No entanto, devido à variação de setores, atividades e características das MPE's, de acordo com Maiti et al. (2020), é necessário o uso de técnicas e ferramentas que possibilitem uma modelagem e parametrização de cada problemática. Na visão destes autores, o uso de modelos de Linear Programming (LP) se apresenta como vantajoso por ser de baixa complexidade em termos de tempo de execução para que MPE's obtenham vantagem competitiva.

Nesse sentido, Cvetkoska (2016) afirma que o avanço proporcionado por modelos de LP é a simplicidade e a versatilidade baseada em recursos. Algo desejável para MPE's em seu gerenciamento e sustentabilidade do negócio, especialmente em seus estágios iniciais do ciclo de vida, complementa esta autora. Na opinião de Bagshaw (2019) e Wu et al. (2019), uma 
Citação (APA): Santos., D. V., Gomes, K. Q., Rocha, A. R., Zatta, F. N., Mattos, A. L., \& Goncalves, W. (2021). Mathematical Programming como ferramenta gerencial em micro e pequenas empresas: uma proposta para apoio à decisão no setor de vestuário. Brazilian Journal of Production Engineering, 7(3): 56-70.

característica significativa da LP é que, matematicamente, o modelo proposto pode ser convertido em modelos de Mixed-integer linear programming ou de Stochastic Linear Programming e, assim, passa a atender a variação temporal - algo comum às pequenas empresas.

Devido à plurivalência e adaptabilidade a diversos contextos e cenários, a LP foi selecionada como diretriz à resolução de um problema cotidiano de pequenas empresas por modelagem matemática. Para Dantzig (1955), a LP é usada, em geral, para auxiliar a tomada decisão sobre a combinação ideal de elementos ou variáveis e a alocação de recursos limitados, os quais são simulados a partir da geração de lucros máximos ou custos mínimos.

Em geral, um modelo de LP é definido por meio de uma função objetivo, que é estabelecida para auxiliar a análise de um determinado problema e, ainda, garantir que todos os elementos importantes deste problema sejam considerados (Maiti et al., 2020; Boyd et al., 2004). Uma característica essencial da função objetivo é a consideração de sua representação em duas ou mais partes (Dantzig, 1955).

A operacionalização da simulação da otimização de um problema compreende a formulação de uma função de objetivo, a qual é delimitada por um conjunto de restrições (Boyd et al., 2004). No entanto, conforme Wu et al. (2019) essa função, ao considerar o resultado desejado, envolve a triangulação de variáveis que, por conseguinte, ponderam a maximização dos resultados desejáveis, quais sejam, lucro, conveniência, receita, resultados do colaborador, eficiência da máquina, etc. ou a minimização de resultados adversos como: custo, preço, taxa, distância percorrida, tempo de execução, peso, etc.

Dantzig (1955) destaca que em um problema LP, os coeficientes da função objetivo necessitam ser identificados de acordo com um diagnóstico prévio do problema a ser analisado. Em relação a isso, este autor enfatiza que o tomador de decisão precisa conhecer quais variáveis estão envolvidas no problema, caracterizando o que se deseja de cada uma delas. Adicionalmente, Bagshaw (2019) menciona que as restrições precisam ser identificadas em termos de limitações. De maneira geral, a função objetivo apresenta a seguinte configuração (Equação 1):

Max. ou Min. $Z=a_{1} x_{1}+a_{2} x_{2}+a_{3} x_{3}+\cdots+a_{n} x_{n}$

Em que: $a_{1}, a_{2}, a_{3}, \cdots, a_{n}$, são coeficientes da função objetivo e, $x_{1}, x_{2}, x_{3}, \cdots, x_{n}$, são as variáveis de decisão.

Desta forma, as restrições são delimitações que norteiam decisões ou objetivos (Maiti et al., 2020). Isso, por conseguinte, é ratificado por Cvetkoska (2016) ao assegurar que essas restrições não excedam os recursos à disposição. Para esta autora, essas restrições ocorrem essencialmente em duas dimensões: as funcionais que estão relacionadas a requisitos de entrada e ao atendimento aos limites dos recursos disponíveis - Equação 2, e as de quantidade (Equação 3).

Sujeito a:

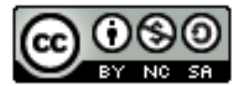




$$
\begin{aligned}
& \left\{\begin{array}{l}
b_{11} x_{1}+b_{12} x_{2}+b_{13} x_{3}+\cdots+b_{1 n} x_{n}+\leq k_{1} \\
b_{21} x_{1}+b_{22} x_{2}+b_{23} x_{3}+\cdots+b_{2 n} x_{n}+\leq k_{2} \\
b_{31} x_{1}+b_{32} x_{2}+b_{33} x_{3}+\cdots+b_{3 n} x_{n}+\leq k_{3} \\
b_{m 1} x_{1}+b_{m 2} x_{2}+b_{m 3} x_{3}+\cdots+b_{m n} x_{n}+\leq k_{m}
\end{array}\right. \\
& x_{1}, x_{2}, x_{3}, \cdots, x_{n} \geq 0
\end{aligned}
$$

Em que: $b_{11}, b_{12}, \cdots, b_{21}, b_{22}, \cdots, b_{31}, b_{32}, \cdots, b_{m 1}, b_{m 2}, \cdots, k_{1}, k_{2}, k_{3} \cdots k_{m}$ são os coeficientes de entrada e, $b_{i j}, i=1,2,3, \cdots, m ; j=1,2,3, \cdots, n$, sendo $i$, a quantidade de recurso consumido por 1 unidade de atividade $j$.

Usualmente, segundo Boyd et al. (2004), na resolução de problemas de LP, os contextos que requerem a maximização de elementos têm restrições menores ou iguais $(\leq)$ a determinados valores limitantes, enquanto os problemas de minimização possuem restrições maiores ou iguais $(\geq)$. Por esse motivo, variáveis de decisão são o foco principal da função objetivo, sendo essencial conhecer suas unidades de mensuração, homem-hora, quantidade a ser produzida, quilos, elementos de custo, e assim por diante, como também são a base para as restrições (Bagshaw, 2019; Dantzig, 1955).

\section{Abordagem Metodológica}

Nesta seção, apresentamos a abordagem utilizada no caso que serviu para examinar, avaliar e contribuir para elaboração do modelo Linear Programming (LP) proposto (Equações 4 e 5). Para tanto, foram consideradas que as decisões tomadas no problema estudado não são independentes umas das outras devido a requisitos de sincronização organizacional. Por esse motivo, a interdependência dessas decisões nos permite conhecer o valor das variáveis de decisão de nível superior no primeiro estágio (função objetivo) e, em seguida, otimizar o problema de nível inferior restante no segundo estágio (restrições), fixando as variáveis de decisão de nível superior.

A realização deste estudo de caso teve por base um survey e uma modelagem matemática, ambos direcionados à elaboração de uma solução que auxilie à tomada de decisão em pequenas empresas. Essa realização foi efetivada por meio de 5 etapas (Figura 2). A unidade de pesquisa considerada neste estudo de caso compreendeu uma pequena empresa do setor de vestuário, a qual possui 10 colaboradores e uma representatividade significativa na região, localizada em uma cidade da região do Rio Doce (Minas Gerais). Essa empresa foi selecionada por estar situada em uma região a qual necessita elevar sua atratividade e soluções que apoiem sua cadeia produtiva (Cunha et al., 2017). 
Figura 2. Síntese operacional da abordagem metodológica

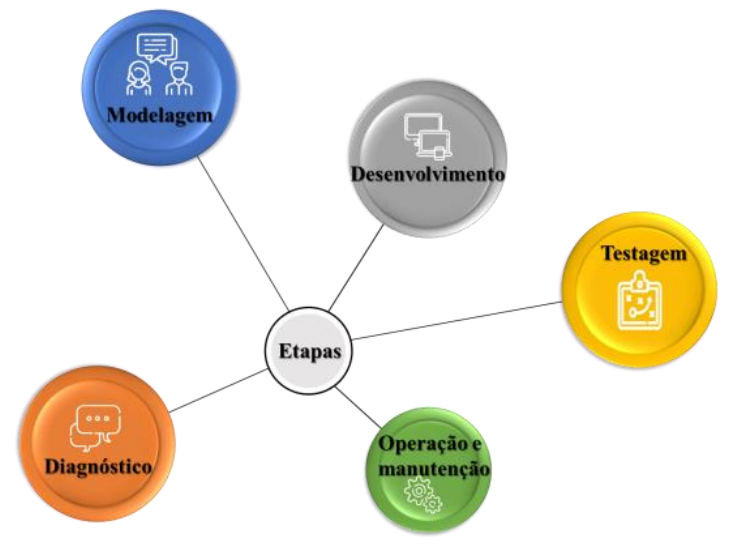

Fonte: Autores.

$\mathrm{Na}$ primeira etapa da abordagem realizou-se um diagnóstico a partir de uma sessão de brainstorming com o gestor e três colaboradores da área operacional da empresa (Akaeze, 2016). Nessa sessão foram obtidos dados que subsidiaram a elaboração do instrumento de coleta. Em seguida, após elaborar o instrumento, este foi aplicado ao gestor e a todos os colaboradores da empresa por meio de questionário eletrônico, no período de 02 a 26/07/2016, sendo levantados elementos que caracterizaram o problema da empresa (Cvetkoska, 2016).

Em seguida, a partir do conhecimento do problema a ser resolvido e dos elementos que o compõe, foi elaborada uma modelagem matemática (Maiti et al., 2020). Na elaboração dessa modelagem foi considerada uma interação com stakeholders e usuários da solução, tanto em termos de coleta de dados quanto na usabilidade das informações geradas. Assim, o desenvolvimento da solução ocorreu por meio de linguagem de programação primária (LINGO 17) - script, em que a modelagem elaborada foi implementada. Esse desenvolvimento considerou as particularidades do setor e da empresa, além do conhecimento matemático necessário para sua utilização.

Posteriormente, por intermédio de teste do script, foi possível verificar a funcionalidade, usabilidade, velocidade de processamento, precisão das operações e da integração de processos, confiabilidade, além da facilidade de portabilidade para outro ambiente computacional e funcionamento apropriado. Após a testagem da solução, esta foi apresentada à unidade de pesquisa para entrar em operação no seu cotidiano. Para tanto, foi realizado um treinamento em que todo o ciclo operacional e de manutenção foram apresentados.

O processamento estatístico do survey foi realizado com o emprego do software SPSS (Statistical Package for the Social Science) Statistics Desktop 23.0 - versão trial, a fim de contribuir para minimização de erros. Desta forma, a análise de dados foi antecedida de preparo inicial, em que missing values (valores em falta) e outliers (valores atípicos) foram verificados para evitar possíveis discrepâncias. Para auxiliar a modelagem matemática, implementação, testagem e manutenção da solução de LP foi utilizado o solver LINGO 17 versão trial.

A verificação da confiabilidade interna da escala utilizada no instrumento de coleta foi examinada por meio do alfa de Cronbach $\left(C_{\alpha}\right)$, em que um valor do coeficiente igual ou superar 
a 0,7 indica consistência adequada (Taber, 2018). Com relação a ocorrência de outliers foi utilizado o intervalo $-2,5<Z<2,5$, que segundo Aronne et al. (2020) e Brudvig et al. (2019) para amostras de até 100 elementos se apresenta como eficiente na detecção dessas anomalias.

\section{RESUltados}

O conjunto de dados utilizados neste estudo de caso foi primário. Isso ocorreu porque em diversos casos MPE’s do setor de vestuário não dispõem de registros organizados que permitam uma análise ampliada e transversal de suas operações. Assim, com este pensamento, foi iniciada a coleta de dados na pequena empresa do setor de vestuário localizada em uma cidade da região do Vale do Rio Doce (Minas Gerais), a qual está no mercado desde 2004 e, que, distribui seus produtos regionalmente.

A partir da sessão de brainstorming - realizada com o gestor e os três colaboradores da área operacional da empresa - foi possível o entendimento da problemática a ser abordada. Logo, ficou evidenciado que a empresa tem enfrentado problemas que envolvem a sua capacidade produtiva mostrando-se ineficiente quanto à utilização de recursos (materiais e patrimoniais) e, além disto, carência de planejamento e controle da produção, o que tem contribuído para o atraso de entregas junto aos clientes. Por esses motivos, o gestor da empresa relatou que almeja tomar decisões que aprimorem o desempenho, a fim de aperfeiçoar seus resultados e, com isso, elevar seu nível de competitividade frente ao mercado.

Isto posto, foi levantada a necessidade de maximizar o lucro da empresa, assim como, informações referentes à quantidade de recursos disponíveis, horas de trabalho, demanda e lucro por unidade produzida. A amostragem foi composta por todos os colaboradores, os quais responderam ao instrumento de coleta de dados. A consistência interna desse instrumento foi atestada ao se obter um $C_{\alpha}$ igual a 0,83 . Ao realizar análise dessa amostragem verificou-se ausência de outliers e de missing values.

Desta forma, segundo os dados levantados, foi detectado que a empresa produz e comercializa cinco tipos diferentes de produtos (Tabela 1), com uma jornada de trabalho de 44 horas semanais, desempenhada por 10 colaboradores, disponibilidade de 2520 metros de tecido, e, uma capacidade produtiva de 550 peças por semana. Assim, o processo produtivo da empresa foi caracterizado considerando uma unidade produzida de cada item do mix disponível no momento do diagnóstico.

Tabela 1. Parâmetros da produção

\begin{tabular}{lccc}
\hline \multicolumn{1}{c}{ Produto } & Lucro/unidade $(\mathbf{R} \$)$ & Tecido $(\mathbf{m})$ & Mão de Obra $(\mathbf{m i n})$. \\
\hline Camisa polo & 11,80 & 0,80 & 12 \\
Camisa Gola Careca & 12,98 & 0,70 & 5 \\
Calça & 11,80 & 1,20 & 37,50 \\
Avental & 12,98 & 0,60 & 16 \\
Touca & 5,90 & 0,40 & 4 \\
\hline
\end{tabular}

Fonte: Autores.

O ciclo produtivo anual da empresa considera um período de 8 meses, o qual subsidia a elaboração do planejamento operacional, sendo a produtividade média requerida para delimitação deste ciclo. Após a definição dessa informação, foram levantados ciclo produtivos 
considerando os últimos cinco anos (Tabela 2). E, por solicitação do gestor da empresa, foi estimado um cenário pessimista, verificado a partir de uma Média Geométrica $\left(M_{G}\right)$ das demandas desses anos. E, deste modo, foi visualizada a tendência central desse conjunto de dados (Mohd Tajuddin et al., 2017).

Tabela 2. Previsão de demanda

\begin{tabular}{|c|c|c|c|c|c|c|}
\hline \multirow{2}{*}{ Produto } & \multicolumn{5}{|c|}{ Produtividade (Un.) } & \multirow{2}{*}{$M_{G}(\mathbf{U n} \mathbf{.})$} \\
\hline & 2015 & 2016 & 2017 & 2018 & 2019 & \\
\hline Camisa Polo & 30 & 50 & 65 & 70 & 90 & 57 \\
\hline Camisa Gola Careca & 85 & 100 & 130 & 140 & 180 & 123 \\
\hline Calça & 35 & 50 & 65 & 70 & 90 & 59 \\
\hline Avental & 17 & 20 & 26 & 28 & 36 & 25 \\
\hline Touca & 15 & 20 & 26 & 28 & 36 & 24 \\
\hline
\end{tabular}

Fonte: Autores.

A modelagem matemática do problema de LP considerou a existência de uma interação entre a empresa, stakeholders e usuários da solução (Maiti et al., 2020), sendo formulada a função objetivo em decorrência da coleta de dados, considerando ainda a usabilidade das informações a serem geradas (Equação 4).

Max. $L=11,8 x_{1}+12,98 x_{2}+11,8 x_{3}+12,98 x_{4}+5,9 x_{5}$

Em que: $x_{1}$ é a quantidade de Camisa Polo a ser produzida; $x_{2}$ é a quantidade de Camisa Gola Careca; $x_{3}$ é a quantidade de Calça; $x_{4}$ é a quantidade de Avental e $x_{5}$ é a quantidade de Touca.

Analogamente, a partir dos artefatos disponíveis, tornou-se necessária a delimitação das restrições, pois, deste modo, além da prevenção de excessos, possibilita-se a adoção de um planejamento racionalizado de recursos (Cvetkoska, 2016). À vista disso, as restrições da função objetivo foram formuladas considerando a dimensão funcional dos elementos que a compõe, assim como o atendimento aos limites e quantidades dos recursos disponíveis (Equação 5).

Sujeito a:

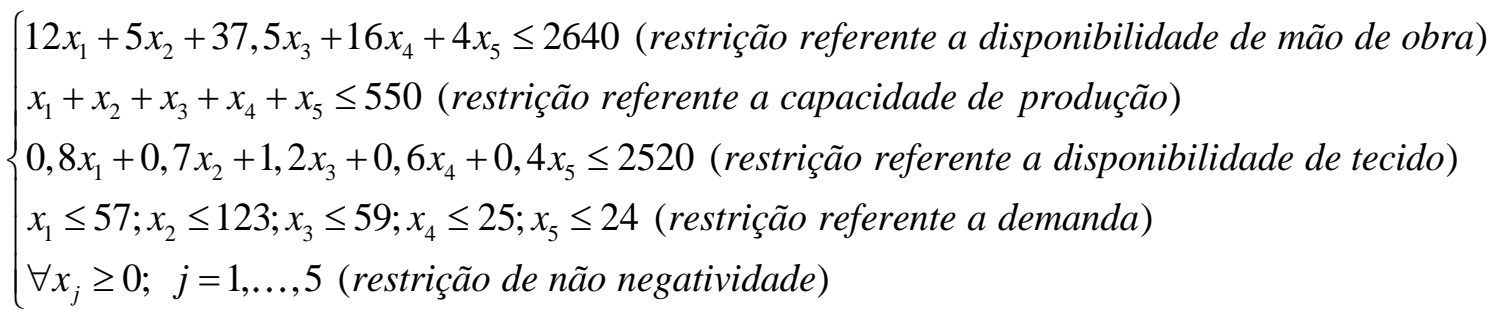

Posteriormente, o desenvolvimento do script da solução foi realizado considerando a aplicabilidade para a empresa do caso em estudo e, também, de forma que possa ser adaptado ou reformulado para empresas de mesmo porte no segmento de vestuário (Figura 3). Adicionalmente ao script, foram elaboradas orientações e diretrizes quanto ao uso.

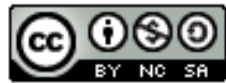


Figura 3. Inserção da modelagem matemática no solver

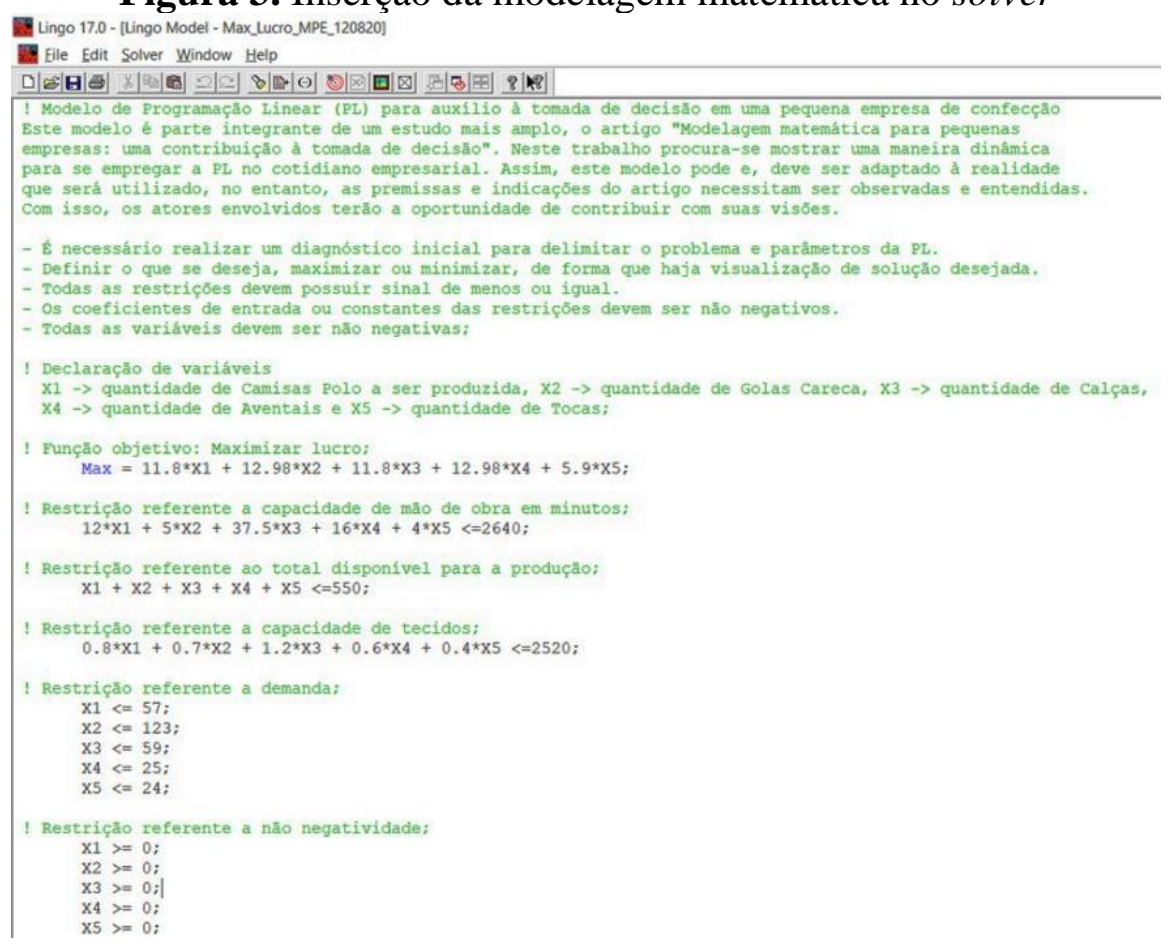

Fonte: Autores.

Assim, o modelo foi simulado e testado em um notebook DELL Inspiron 15 3000, equipado com processador Intel ${ }^{\circledR}$ Core $^{\mathrm{TM}} \mathrm{i} 7-8565 \mathrm{U}\left(1.8 \mathrm{GHz}\right.$ até $4.6 \mathrm{GHz}$, cache de $8 \mathrm{MB}$, quad-core, $8^{\mathrm{a}}$ geração), Windows 10 Home Single Language, de 64 bits - em Português (Brasil), Placa de vídeo dedicada AMD Radeon ${ }^{\mathrm{TM}} 520$ com 2GB de GDDR5, Memória de 8GB (1x8GB), DDR4, 2400MHz; Expansível até 16GB (1 slot soDIMM, sem slot livre) e SSD de 128GB + HD de 1TB 5400 RPM (Figura 4).

Figura 4. Dados de saída do solver

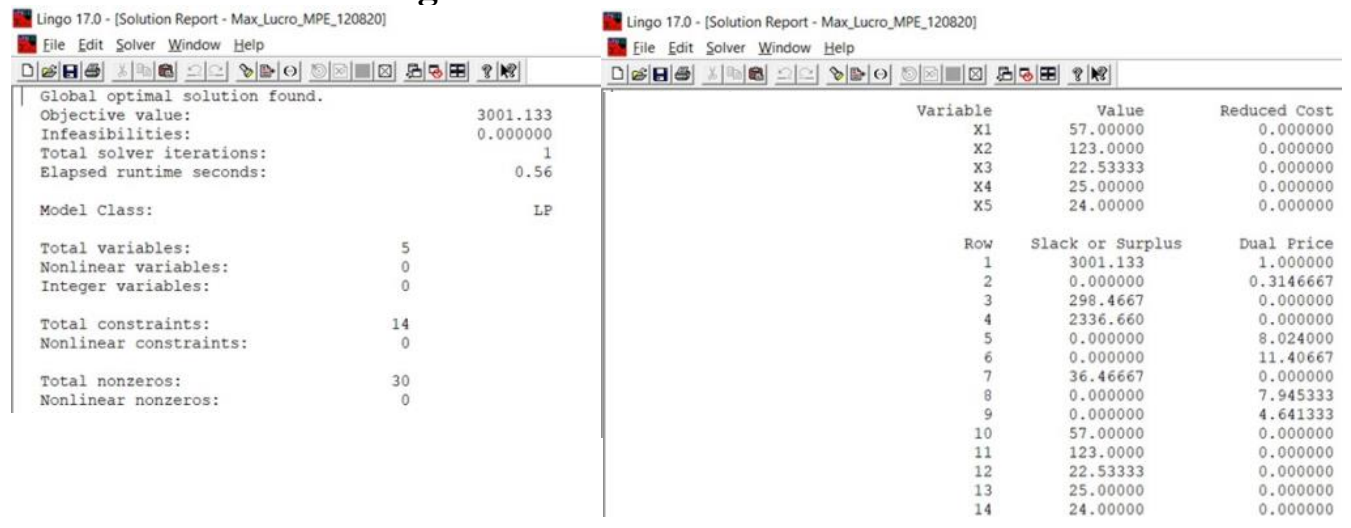

Fonte: Autores.

Ao realizar a simulação, foi obtido como solução ótima o valor de $\mathrm{R} \$ 3.001,13$, que significa o lucro máximo da empresa, com um processamento realizado em 15 milésimos de segundo, utilizando $29 \%$ da memória do notebook e uma única inteiração, considerando o diagnóstico realizado, ou seja, 5 variáveis $\left(x_{1} ; x_{2} ; x_{3} ; x_{4}\right.$ e $\left.x_{5}\right)$ e 14 restrições. Esse resultado compreende o 
lucro obtido com a produção de 57 unidades de Camisa Polo $\left(x_{1}\right), 123$ Camisas Gola Careca ( $\left.x_{2}\right), 22$ Calças $\left(x_{3}\right), 25$ Aventais $\left(x_{4}\right)$ e 24 Toucas $\left(x_{5}\right)$.

Vale ressaltar que a solução ótima utiliza 99,24\% da capacidade de mão de obra, 45,64\% da produção e $12 \%$ de tecido. Esses números aparentemente sugerem um desbalanço da produção atual da empresa. Por outro lado, embora este estudo de caso não tenha pretendido investigar e impactar no nível de balanceamento da linha de produção (Nallusamy, 2016), vale destacar que o planejamento e controle somados à uma estratégia de produção, permitem, dentre outros benefícios, a otimização do uso de recursos, que por sua vez afeta diretamente esse balanceamento.

\section{CONSIDERAÇÕES FINAIS}

Integrar tecnologia, medidas de flexibilidade e processos que possam ser inovados na gestão de operações é uma tendência do mundo moderno. De maneira especial hoje em dia a flexibilidade é algo que necessita estar presente no cotidiano da gestão empresarial, uma vez que o comportamento do mercado tem mudado rapidamente e, com isso, toda a economia acaba assumindo esse comportamento de incerteza. Por estes motivos, a adaptabilidade às adversidades do mercado pode ser considerada como uma das principais ferramentas de sobrevivência para a maioria das micro e pequenas empresas.

Com esta visão, neste estudo de caso examinamos com sucesso diversos tipos de produtos do mix, recursos disponíveis, quantidades e custos de produção de uma pequena empresa do setor de confecção. Sugerimos uma solução ideal usando dados secundários coletados dos registros da empresa, por meio de um problema de Linear Programming (LP), formulado com modelagem matemática usando o solver LINGO.

A solução revelou que o gestor da empresa deve se concentrar mais na produção de camisa Gola Careca e Polo, enquanto os outros tipos devem ser avaliados e produzidos de acordo com a estratégia de posicionamento de mercado, pois suas contribuições devem ser acompanhadas com rigor mais elevado, a fim de atingir o lucro mensal máximo desejado.

Desta forma, teorias, análises e fórmulas são estudadas, aplicadas e demonstradas com exemplo prático. Incertezas nas MPE's são desafios para as operações, especialmente quando se trata de orçamento, planejamento, gestão e controle. Por esse motivo, compreender estas incertezas auxilia a minimização de riscos, sendo importante para evitar resultados abaixo do ideal. $\mathrm{O}$ modelo determinístico apresentado neste estudo de caso é ferramenta eficaz para lidar com questões de gerência empresarial e da produção, sobretudo com relação a alocações ótimas de recursos, o qual pode e deve ser adaptado para diferentes cenários e condições.

Destarte, pesquisas futuras podem expandir o escopo deste estudo para incluir outros interconectores como colaboradores, clientes e fornecedores. Por fim, nossa abordagem de modelagem pode ser enriquecida com métodos multicritério de auxílio à tomada de decisão, como o Analytic Hierachy Process (AHP) e o Elimination et Choix Traduisant la Realité (ELECTRE), a fim de ampliar horizontes e fornecer considerações para realizar um planejamento flexível. E, desta forma, propor uma ferramenta de simulação e desenvolvimento de cenários, a qual pode ser de uso direto por formuladores de políticas, elevando a consideração de suas prioridades.

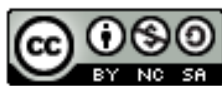




\section{Agradecimentos}

Laboratório de Pesquisa Operacional Logística e Transportes (POLT) da Universidade Federal do Espírito (UFES) / Centro Universitário Norte do Espírito Santo (CEUNES).

\section{REFERÊNCIAS}

Akaeze, C. O. (2016). Exploring Strategies Required for Small Business Sustainability in Competitive Environments. [Tese de Doutorado, Walden University] WU Campus Repository. Recuperado de https://scholarworks.waldenu.edu/cgi/viewcontent.cgi?article=2976\&context=dissertations

Aronne, A., Grossi, L., \& Bressan, A. A. (2020). Identifying outliers in asset pricing data with a new weighted forward search estimator. Revista Contabilidade \& Finanças, 31(8), 458-472. https://dx.doi.org/10.1590/1808-057x201909620

Associação Brasileira da Indústria Têxtil e de Confecção. (2020). Enquete com os empresários do setor têxtil e confecção: acompanhamento sobre os impactos do Coronavírus: São Paulo: 2020. Recuperado de https://www.abit.org.br/uploads/arquivos/SEMANA\%202327\%20impactos\%20Covid.19\%20 pptx.pdf

Associação Brasileira da Indústria Têxtil e de Confecção. Síntese do Comércio Exterior Brasileiro no setor têxtil e de confecção: São Paulo: 2021. Recuperado de https://www.abit.org.br/uploads/arquivos/S\%C3\%ADntese\%20COMEX\%20BR\%20mar\%C3 \%A7o\%2021.pdf

Bagshaw, K. B. (2019). A Review of Quantitative Analysis (QA) in Production Planning Decisions Using the Linear Programming Model. American Journal of Operations Research, 9, 255-269. https://doi.org/10.4236/ajor.2019.96017

Birbirenko, S. (2020). Strategic development as a factor in ensuring the economic stability of a modern market enterprise. Three Seas Economic Journal, 4(1), 18-23.

Boyd, S., Boyd, S. P., \& Vandenberghe, L. (2004). Convex optimization. Cambridge university press.

Brito, R. P., \& Brito, L. A. L. (2012). Vantagem competitiva e sua relação com o desempenho. Revista de Administração Contemporânea, 16(3), 360-380. https://doi.org/10.1590/S141565552012000300003

Brudvig, S., Brusco, M. J., \& Cradit, J. D. (2019). Joint selection of variables and clusters: recovering the underlying structure of marketing data. Journal of Marketing Analytics, 7(1), 01-12. https://doi.org/10.1057/s41270-018-0045-7

Confederação Nacional da Indústria. (2017). O setor têxtil e de confecção e os desafios da sustentabilidade: Brasília: 2017. Recuperado de https://bucket-gw-cni-staticcmssi.s3.amazonaws.com/media/filer_public/bb/6f/bb6fdd8d-8201-41ca-

981ddeef4f58461f/abit.pdf

Cunha, D. M., Gomes, M. F. A., \& Fernandes, D. M. (2017). Microrregião de Governador Valadares (MG): a dinâmica de seus fluxos migratórios. Espaço em Revista, 19(1), 98-115. https://doi.org/10.5216/er.v19i1.47338

Cvetkoska, V. (2016). A survey of the use of operational research in decisions made by micro, small and medium-sized enterprises in Macedonia. Croatian Operational Research Review, 7(2), 349-365. https://doi.org/10.17535/crorr.2016.0024

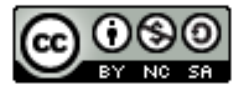


Dantzig, G. B. (1955). Linear programming under uncertainty. Management science, 1(3-4), 197-206. https://doi.org/10.1287/mnsc.1.3-4.197

Filleti, J. P, \& Boldrin, R. (2020). A indústria têxtil no Brasil: um modelo econométrico analisando a hipótese de desindustrialização setorial. Economia e Sociedade, 29(3), 861-890. https://doi.org/10.1590/19823533.2020v29n3art08

Freddi, E. (2019). Um estudo sobre programação linear e aprendizagem baseada em problemas. [Dissertação de Mestrado, Instituto de Matemática Estatística e Computação Científica, Universidade Estadual de Campinas]. http://repositorio.unicamp.br/handle/REPOSIP/334916

Gamboa, U. M. R., Maciel, V. F., Vendruscolo, B. D., \& Silva, H. (2020). Os efeitos potenciais do regime tributário competitivo para confecção (RTCC): Uma aplicação de vetores autorregressivos (VAR). Revista de Economia Mackenzie, 17(1), 146-164. https://doi:105935/1808-2785/rem.v17n1p.146-164

Godinho, I. P., \& Corso, L. L. (2019). Aplicação da Programação Linear para otimizar o mix de produtos em uma empresa de confecção. Scientia cum Industria, 7(2), 83-87. http://dx.doi.org/10.18226/23185279.v7iss2p83

Gomes, J., Mariz, E. C., Silva, A. M., \& Kawamoto, L. T. J. (2019). Programação linear aplicada à mobilidade urbana: análise do trajeto da residência à escola de estudantes universitários. Revista Eniac Pesquisa, 8(2), 280-297. https://doi.org/10.22567/rep.v8i2.581

Hamidi, S., Zandiatashbar, A., \& Bonakdar, A. (2019). The relationship between regional compactness and regional innovation capacity (RIC): Empirical evidence from a national study. Technological Forecasting and Social Change, 142, p. 394-402. https://doi.org/10.1016/j.techfore.2018.07.026

Instituto de Pesquisa Econômica Aplicada. IPEA Nota técnica $n^{o}$ 63: Socorro governamental às pequenas unidades produtivas frente à atual pandemia: Brasília: 2020. Recuperado de http://repositorio.ipea.gov.br/handle/11058/10033

Kapitonov, I. A., Taspenova, G. A., Meshkov, V. R., \& Shulus, A. A. (2017). Integration of small and middle-sized enterprises into large energy corporations as a factor of business sustainability. International journal of energy economics and policy, 7(2), p. 44-52. http://www.econjournals.com/index.php/ijeep/article/download/4040/2739

Maciel, V. F., Fronzaglia, M. L., Silva, A. L. P., Silva, H., \& Orlandi, K. W. (2019). Cadeia têxtil-confecção: competitividade e intervenção governamental na segunda década do século XXI no Brasil. In: Vartanian, P. R., Maciel, V. F. (orgs.). Estudos econômicos setoriais: Máquinas e equipamentos, ferrovias, têxtil e calçados (pp. 129-168). Blucher Open Access.

Maiti, M., Krakovich, V., Shams, S. R., \& Vukovic, D. B. (2020). Resource-based model for small innovative enterprises. Management Decision, 58(8), 1525-1541. https://doi.org/10.1108/MD-06-2019-0725

Mohd Tajuddin, R. B., Hashim, S. F. B., \& Zainol, A. S. B. (2017). The role of brand identity in creating resilient small enterprises (SME's) in fashion industry. International Journal of Supply Chain Management, 6(2), 140-146. Recuperado de http://ijisscm.bsne.ch/ojs.excelingtech.co.uk/index.php/IJSCM/article/download/1469/1469-5677-1$\underline{\text { PB.pdf }}$

Nallusamy, S. (2016). Productivity enhancement in a small scale manufacturing unit through proposed line balancing and cellular layout. International Journal of Performability Engineering, 12(6), 523-534. 

Journal of Production Engineering, 7(3): 56-70.

Pavão, J. A., \& Camacho, R. R. (2018). Estratégia, tecnologia e inovação em empresas do setor de confecções: uma investigação em um arranjo produtivo local. Caderno de Administração, 26(1), 98-114.

Pereira, F. C. M, Carvalho, R. B., \& Quintão, A. A. (2019). Uso da inteligência competitiva por micro, pequenas e médias empresas: estudo de caso nas empresas associad as à ACITA, Itabira (MG), Brasil. Revista Inteligência Competitiva, 9(3), 100-122. https://doi.org/10.24883/ric.v9i3.334

Porter, M. E. (1996). Competitive advantage, agglomeration economies, and regional policy. International regional science review, $19(1-2), \quad 85-90$. https://doi.org/10.1177/016001769601900208

Santos, J. N., \& Vallim, C. R. (2020). Programação linear na otimização de mix de serviços: um estudo de uma empresa de hotelaria. Anais do XXVII Congresso Brasileiro de Custos. Associação Brasileira de Custos.

Serviço Brasileiro de Apoio às Micro e Pequenas Empresas. (2014). Participação das Micro e Pequenas Empresas na Economia Brasileira: Brasília: 2014. Recuperado de https://www.sebrae.com.br/Sebrae/Portal\%20Sebrae/Estudos\%20e\%20Pesquisas/Participacao \%20das\%20micro\%20e\%20pequenas\%20empresas.pdf

Serviço Brasileiro de Apoio às Micro e Pequenas Empresas. (2020). Micro e pequenas empresas geram 27\% do PIB do Brasil: Brasília: 2020. Recuperado de https://www.sebrae.com.br/sites/PortalSebrae/ufs/mt/noticias/micro-e-pequenas-empresasgeram-27-do-pib-do-brasil,ad0fc70646467410VgnVCM2000003c74010aRCRD

Silva, V. P., Nascimento, A. C., Entriel, L. C., Oliveira, S. B., \& Ramos Filho, A. C. (2020). Múltiplas formas de manifestação do conhecimento nas organizações: proposta de framework como abordagem estratégica para alcance da efetividade organizacional. Revista Brasileira de Gestão e Inovação, 8(1), 138-161. https://doi.org/10.18226/23190639.v8n1.07

Siqueira, M. P., Amaro, T. C. F., Quarto, L. C., Souza, S. M. F., \& Quarto, J. V. C. (2019). O processo de formação de preço de uma microempresa do setor têxtil de confecção. Revista Latino-Americana de Inovação e Engenharia de Produção, 7(11), 138-149. http://dx.doi.org/10.5380/relainep.v7i11.66041

Taber, K. S. (2018). The use of Cronbach's alpha when developing and reporting research instruments in science education. Research in Science Education, 48(6), 1273-1296. https://doi.org/10.1007/s11165-016-9602-2.

Turra, E. B., Mioranza, C., \& Coltre, S. M. (2017). A inovação como vantagem competitiva: estudo de caso em uma pequena empresa. Revista Brasileira de Gestão e Inovação, 5(1), 1-22. https://doi.org/10.18226/23190639.v5n1.01

Vasconcelos, P. S., \& Vasconcelos, P. E. A. (2020). Desafios da estratégia empresarial: antes, durante e após a pandemia de 2020. Revista Interdisciplinar de Direito, 18(1), p. 163-182. Recuperado de http://revistas.faa.edu.br/index.php/FDV/article/view/846

Wu, Z., Yang, K., Yang, J., Cao, Y., \& Gan, Y. (2019). Energy-efficiency-oriented scheduling in smart manufacturing. Journal of Ambient Intelligence and Humanized Computing, 10(3), 969-978. https://doi.org/10.1007/s12652-018-1022-x 